\title{
COLLISION MODELING OF SINGLE UNIT IMPACT ABSORBER FOR MECHANICAL SYSTEMS VIBRATION ATTENUATION
}

\author{
Kaouther Chehaibi, Charfeddine Mrad, Rachid Nasri \\ Laboratory of Applied Mechanics and Engineering (LMAI), National Engineering School of Tunis (ENIT), \\ University of Tunis el Manar (UTM), Tunis, Tunisia \\ e-mail: kchehaibi@yahoo.com; charfeddine.mrad@enit.rnu.tn; rachid.nasri@enit.rnu.tn
}

\begin{abstract}
A single unit impact absorber is a ball absorber located in a mechanical system to attenuate its undesirable vibration. The absorber has a free motion constrained by stops. Collisions between the main system and the absorber masses help to dissipate kinetic energy as heat, noise and high frequency vibrations and thus reducing the main system dynamic response. However, the collisions give rise to discontinuity and strong nonlinearity. This work intends to study the effect of the collision modeling on the absorber efficiency. The contact force-based linear viscoelastic model of Hook-Newton, nonlinear elastic model of Hertz, and nonlinear viscoelastic model proposed by Hunt and Crossley are considered. For each case, analytical study is conducted to determine the equations of motion. The system responses are obtained for forced vibration, considering the whole ball motion cycle: left impact-free motion-right impact and following a numerical resolution based on the Newmark method. Finally, comparison of different impact models is made to conclude on the single unit impact absorber performance.
\end{abstract}

Keywords: mechanical system, single unit impact absorber, collision modeling

\section{Introduction}

Impact dampers may be used in many fields to control excessive vibrations of buildings and structures or mechanisms and machines.

Theoretical analysis of impact dampers first began with Lieber and Jensen (1945). They considered each collision as a completely plastic collision. Grubin (1956) introduced the elastic restitution coefficient. He took into account the energy loss during collision and established a theoretical model of a single-degree-of-freedom system equipped with an impact damper under harmonic excitation ( $\mathrm{Lu}$ et al., 2017). The characteristics of the impact damper were investigated in detail when the main vibratory system was vibrated in the direction of gravity and perpendicular to it. Furthermore, application of the impact damper to improve the damping capability of drills was attempted (Ema and Marui, 1996).

A relationship between the coefficient of restitution and the impact damping ratio was developed by Cheng and Wang (2003) and Cheng and Xu (2007). It was shown that the effective reduction of the response amplitude was nearly independent of the number of impacts, but primarily related to the type of collision. Furthermore, the results revealed that the clearance of an effective impact damper should be smaller than twice of the initial displacement of the main mass if the system was stimulated by the initial displacement only.

Bapat and Sankar (1985) studied a single unit impact damper under free and forced vibrations. The effects of mass ratio, coefficient of restitution, and gap size on the free vibrations were determined. In the study of forced motion, they showed the optimum gaps and corresponding displacement amplitude reduction within the resonant frequency range. They proved that the optimum gap at the resonance was not necessarily optimal at other frequencies. 
Vibration attenuation of a mechanical system equipped with an impact absorber is due to collisions between both bodies which cause energy dissipation (Marhadi and Kinra, 2005). Impacts give rise to discontinuity and strong nonlinearity, so the vibro-impact systems can exhibit very rich and complicated dynamic behavior; it is a good testing bench for nonlinear theories (Cheng and $\mathrm{Xu}, 2007$ ). The contact between both bodies is to be investigated closely (Cheng and $\mathrm{Xu}, 2006$ ).

The modeling of the impact damper is basically concerned with the modeling of the impact phenomenon: duration and nature of the impact and the method of analysis. In general, the impact is always modeled either by applying the Hertz theory of contact forces or the concept of coefficient of restitution. The first model was developed by Hertz; the elasto-static theory was used without considering damping to calculate local indentation. Hunt and Crossley (1975) proposed a model based on the Hertz theory of contact with a nonlinear damping force defined in terms of local penetration (Gilardi and Sharf, 2002).

The impacts were modeled using a spring and a damper (Cheng and Wang, 2003).

Since the most convenient modeling of contact is based on the fact that the interaction forces act continuously during the impact, the study in this work is done with only including the contact forces to the equations of motion during the collision. This allows one to fairly describe the actual behavior of the system.

Three contact models are considered in this paper. The linear model of Hooke-Newton defined by linear stiffness and linear viscous damping; a nonlinear model of Hertz defined by only nonlinear stiffness and a nonlinear model of Hunt and Crossley with both nonlinear stiffness and damping.

Hence, for each impact model, using the continuous approach, an analytical study is conducted to determine the equations of motion. It is followed by a numerical resolution to obtain forced responses of the system when it is equipped with the absorber. Comparisons of different impact models are made later.

The Newmark method is used to integrate the differential equations of motion by Matlab software.

\section{Collision models}

The impact between a mechanical system of mass $M=1 \mathrm{~kg}$, stiffness $k=1000 \mathrm{~N} / \mathrm{m}$ and viscous damping $c=0.01 \mathrm{Ns} / \mathrm{m}$ and between a single ball absorber of mass $m=0.3 \mathrm{~kg}$ and clearance $d=2 \cdot 10^{-2} \mathrm{~m}$ is studied. The system is excited with a sinusoidal force $F$ of module $F_{0}=10 \mathrm{~N}$ and pulsation $\omega$. The coefficient of restitution is $e=0.68$.

The impact is firstly considered linear using the Hooke-Newton model, then nonlinear using both Hertz model and Hunt and Crossley model, gradually approaching to the actual impact. All models are composed of known elementary physical models.

\subsection{Hooke-Newton model}

In the Hooke-Newton model, the impact between a single unit impact absorber and a mechanical system is materialized by a linear spring $k_{1}$ for the elastic deformation, and a linear viscous damper $c_{1}$ for the energy dissipation.

Figure 1 shows the system equipped with the absorber in forced vibration according to the Hooke-Newton model.

The contact force is defined as (Gilardi and Sharf, 2002)

$$
F_{c}=k_{1} y+c_{1} \dot{y}
$$




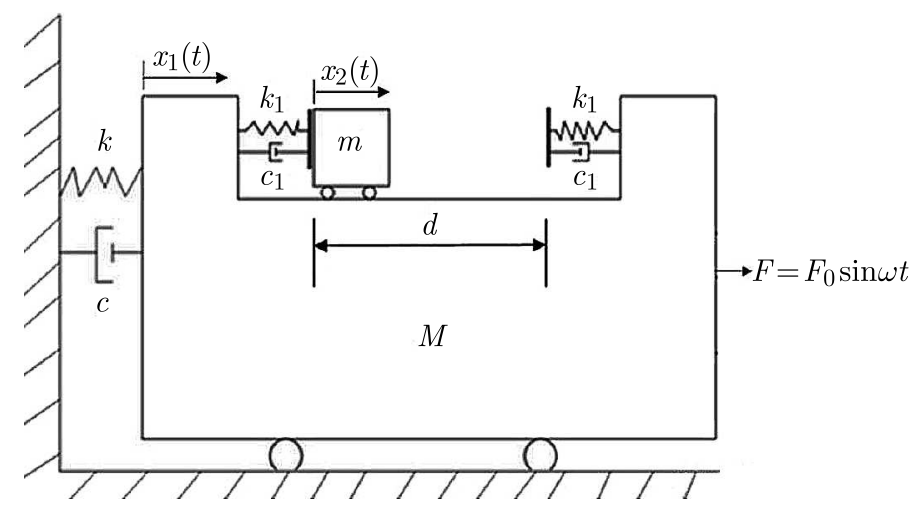

Fig. 1. System equipped with an impact absorber according to the Hooke-Newton model

where $y$ and $\dot{y}$ are, respectively, the relative displacements and relative velocities of masses

$$
y=x_{2}-x_{1} \quad \dot{y}=\dot{x}_{2}-\dot{x}_{1}
$$

$x_{1}$ and $x_{2}$ denote respectively the displacements of the system and the absorber, $\dot{x}_{1}$ and $\dot{x}_{2}$ denote, respectively, the velocities of the system and the absorber.

The equations of motion in forced vibration during the contact between both masses are

$$
\begin{aligned}
& M \frac{d^{2} x_{1}}{d t^{2}}+c \frac{d x_{1}}{d t}+k x_{1}=c_{1} \frac{d y}{d t}+k_{1} y+F(t) \\
& m \frac{d^{2}\left(x_{1}+y\right)}{d t^{2}}+c_{1} \frac{d y}{d t}+k_{1} y=0
\end{aligned}
$$

The collision parameters values for the Hooke-Newton model are given in Table 1.

Table 1. Collision parameters

\begin{tabular}{|c|c|c|}
\hline Parameters & $k_{1}$ & $c_{1}$ \\
\hline \hline Values & $32600 \mathrm{~N} / \mathrm{m}$ & $20.81 \mathrm{Ns} / \mathrm{m}$ \\
\hline
\end{tabular}

The damping constant is determined as (Anagnostopoulos, 2004)

$$
c_{1}=2 \xi \sqrt{k_{1} \frac{m M}{m+M}}
$$

where $\xi$ is expressed as

$$
\xi=-\frac{\ln e}{\sqrt{\pi^{2}+(\ln e)^{2}}}
$$

Figure 2 shows the obtained displacement of the system mass with and without the absorber considering the whole ball motion cycle: left impact-free motion-right impact. The effect of the impact absorber with both a linear spring and a linear damper on the system vibration attenuation is manifested. The main amplitude of vibrations decreases.

\subsection{Hertz model}

The Hertz model is considered. The contact between the system mass and the absorber mass is then defined using a nonlinear stiffness according to the Hertz theory $\left(k_{1}=k_{H z}\right)$, estimated using the material properties: the Young modulus and the Poisson coefficient (Caserta et al., 2016). 


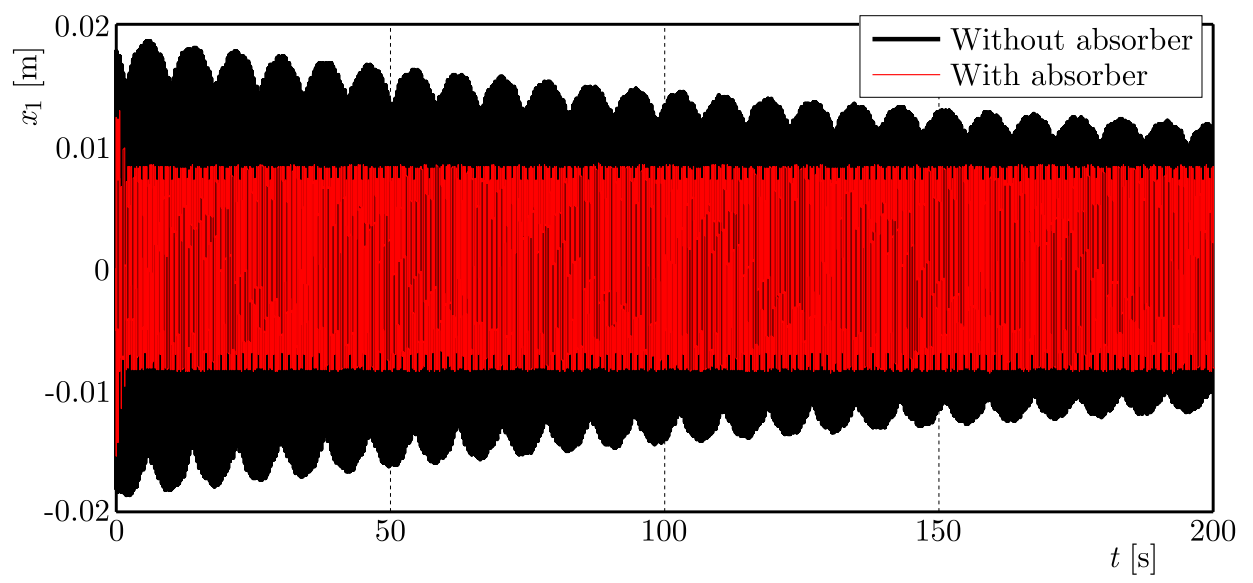

Fig. 2. System displacement according to the Hooke-Newton model

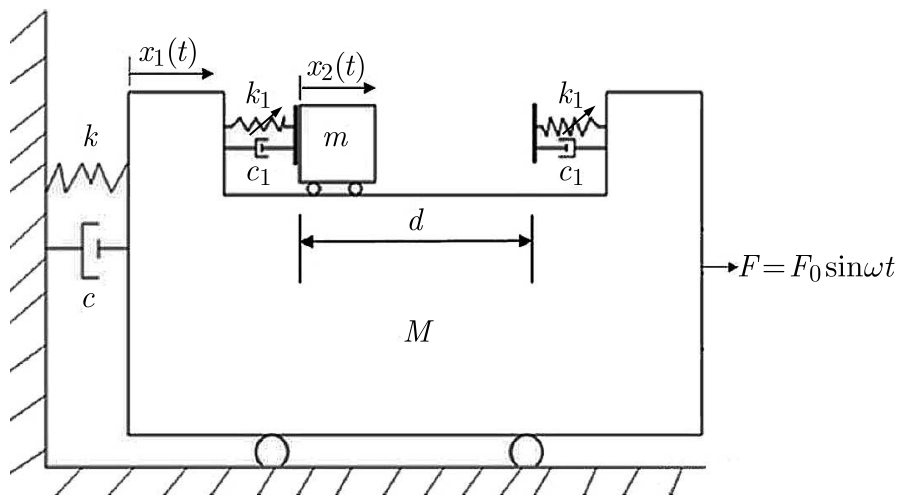

Fig. 3. System equipped with an impact absorber according to the Hertz model

Figure 3 shows the system equipped with the impact absorber in forced vibration according to the Hertz model.

The assumptions for the Hertz model are the following: the deformation is concentrated in the vicinity of the contact zone, the total mass of each body moves with the velocity of its mass center and the movement of the elastic waves is neglected.

The contact force is defined as (Afsharfard and Farshidianfar, 2012)

$$
F_{c}=k_{H z} y^{3 / 2}
$$

where $y$ is the masses relative displacement between the contacting bodies, $k_{H z}$ is the contact stiffness parameter, and $(3 / 2)$ is the nonlinear power exponent that is determined from the material and geometrical properties of the local region of the bodies in contact.

The equations of motion in forced vibration are

$$
\begin{aligned}
& M \frac{d^{2} x_{1}}{d t^{2}}+c \frac{d x_{1}}{d t}+k x_{1}=k_{H z} y^{3 / 2}+F(t) \\
& m \frac{d^{2}\left(x_{1}+y\right)}{d t^{2}}+k_{H z} y^{3 / 2}=0
\end{aligned}
$$

The stiffness constant value for the Hertz model is $k_{H z}=32600 \mathrm{~N} / \mathrm{m}^{3 / 2}$.

Figure 4 shows the obtained displacement of the system mass with and without the absorber considering the whole ball motion cycle: left impact-free motion-right impact. The effect of the absorber with only a nonlinear spring on the system vibration response is pbserved. The main system vibration amplitudes increase instead of decreasing. 


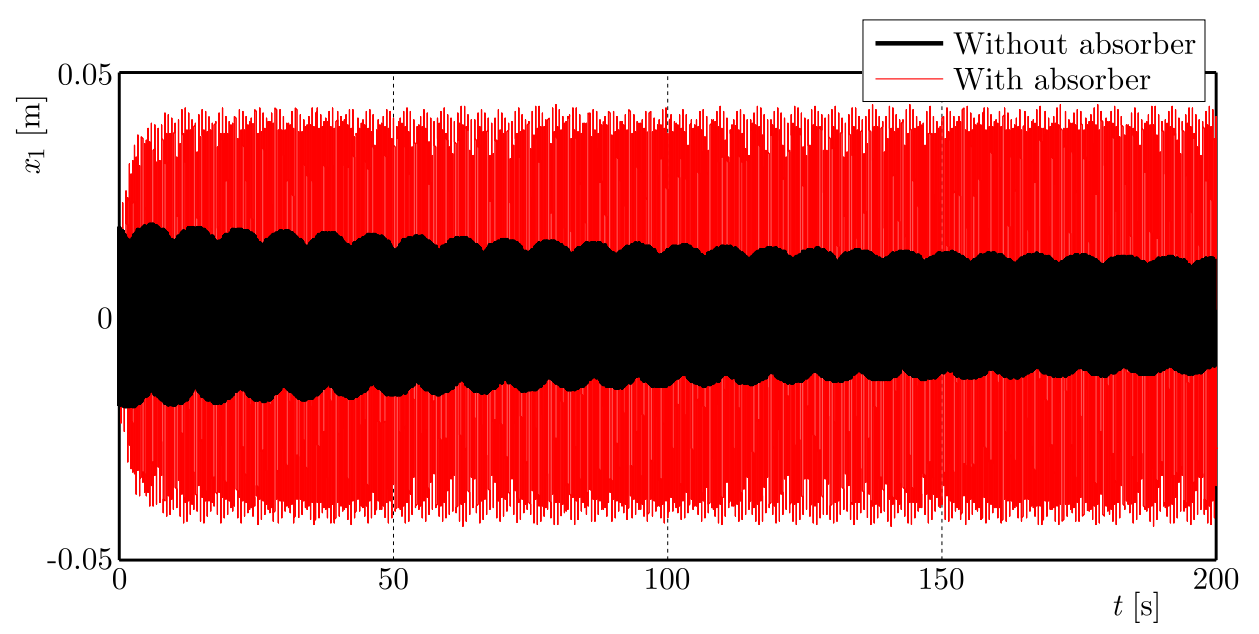

Fig. 4. System displacement according to the Hertz model

\subsection{Hunt and Crossley model}

Hunt and Crossley developed a contact model based on the Hertzian contact model and a non-linear viscous element which accounts for damping and depends on the penetration depth.

The Hunt and Crossley model is used to study the contact between the system and the single unit impact absorber in forced vibration.

Figure 5 shows the system equipped with the absorber in forced vibration according to the Hunt and Crossley model.

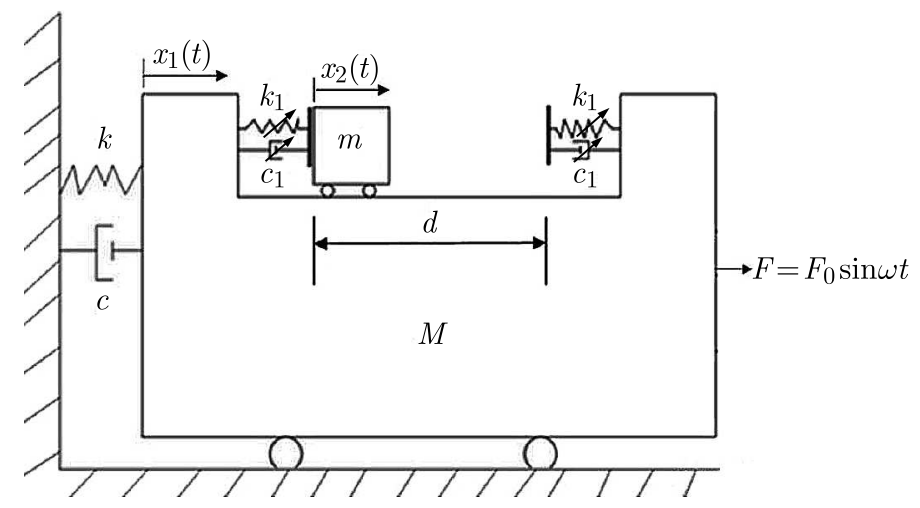

Fig. 5. System equipped with an impact absorber according to the Hunt and Crossley model

The contact force is defined as (Alves et al., 2015)

$$
F_{c}=k_{H z} y^{3 / 2}+c_{1} y^{3 / 2} \dot{y}
$$

where $y$ is the masses relative displacement, $\dot{y}$ is the masses relative velocity (penetration velocity), $k_{H z}$ is the Hertz contact stiffness, $c_{1}$ is the Hunt-Crossley damping coefficient.

The motion equations in forced vibration are

$$
\begin{aligned}
& M \frac{d^{2} x_{1}}{d t^{2}}+c \frac{d x_{1}}{d t}+k x_{1}=k_{H z} y^{3 / 2}+c_{1} y^{3 / 2} \frac{d y}{d t}+F(t) \\
& m \frac{d^{2}\left(x_{1}+y\right)}{d t^{2}}+k_{H z} y^{3 / 2}+c_{1} y^{3 / 2} \frac{d y}{d t}=0
\end{aligned}
$$

The collision parameters values for the Hunt and Crossley model are given in Table 2. 
Table 2. Collision parameters

\begin{tabular}{|c|c|c|}
\hline Parameters & $k_{H z}$ & $c_{1}$ \\
\hline \hline Values & $32600 \mathrm{~N} / \mathrm{m}^{3 / 2}$ & $19560 \mathrm{Ns} / \mathrm{m}^{5 / 2}$ \\
\hline
\end{tabular}

The damping constant is determined as (Alves et al., 2015)

$$
c_{1}=\frac{3 k_{H z}(1-e)}{2 \dot{y}_{0}}
$$

where $\dot{y}_{0}$ is the initial penetration velocity.

Figure 6 shows the obtained displacement of the system mass with and without the absorber considering the whole ball motion cycle: left impact-free motion-right impact. The effect of the impact absorber with both a nonlinear spring and nonlinear damper on the system vibration attenuation is manifestef. The main system vibration amplitudes decrease.

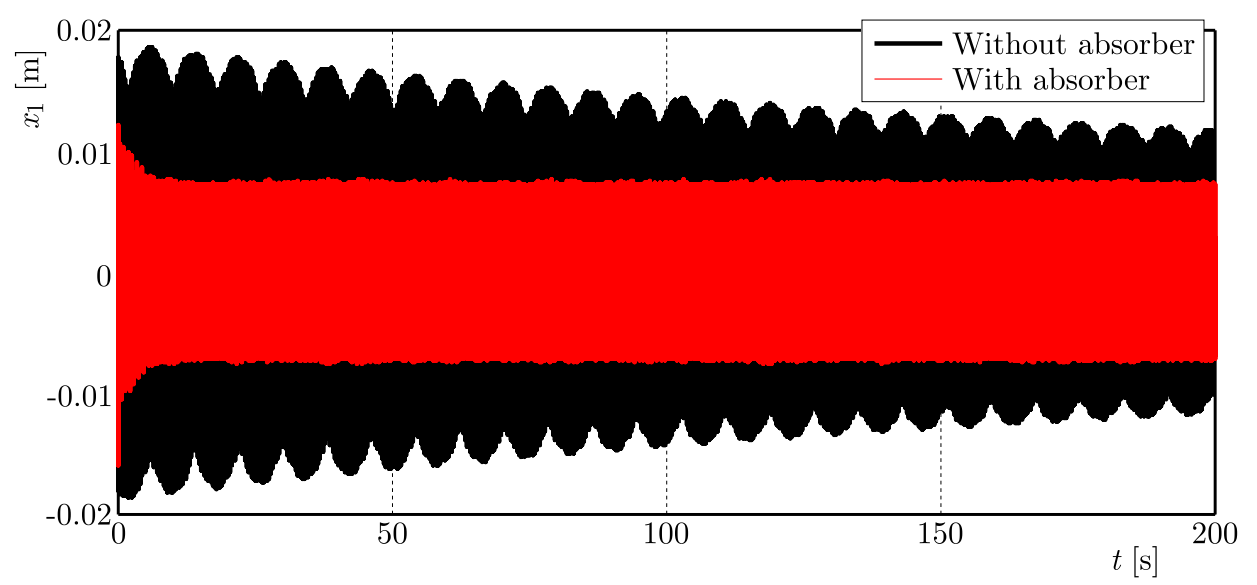

Fig. 6. System displacement according to the Hunt and Crossley model

\section{Models comparison}

The linear Hooke-Newton model does not represent the nonlinearity of the whole contact process and is only suitable for contacts at higher impact velocities (Gilardi and Sharf, 2002). Besides, because the viscous component is active during the whole time of the restitution period (Eq. (2.1)); a negative impact force is observed just before separation, which does not have a physical explanation (Hunt and Crossley, 1975). Moreover, uniform dissipation of energy during the entire contact process is manifested because the coefficient of dissipation is kept constant; which is physically inconsistent. In fact, most of the energy is lost during the approach period, and in the restitution period, the accumulated elastic strain energy is released with only minor energy dissipation. Nevertheless, due to its simplicity, the linear viscoelastic model has been often used.

The nonlinear Hertz model, although it effectively captures the relation between the collision force and deformation, is a perfectly elastic contact-force model so that it does not account for the dissipated energy during collisions due to plastic deformations, friction and local cracking.

The nonlinear model of Hunt and Crossley is best for impacts with a high value of the coefficient of restitution, when the impact typically has lower energy dissipation (Gilardi and Sharf, 2002). An important aspect of this model is that the damping depends on the indentation. This is physically consistent since the contact area increases with deformation and a plastic region is more likely to develop for larger indentations. Another advantage is that the contact 
force has no discontinuities at the initial contact and separation, but it begins and finishes with the correct value of zero.

Hence, the Hooke-Newton model is not too realistic relatively to the actual collision. With the Hertz model, it is not possible to model the compression and restitution phase of the contact. However, the Hunt and Crossley model evaluates the contact process more realistically.

The Hunt and Crossley model is, therefore, considered as the reference in studying the contact between a mechanical system and a single unit impact absorber.
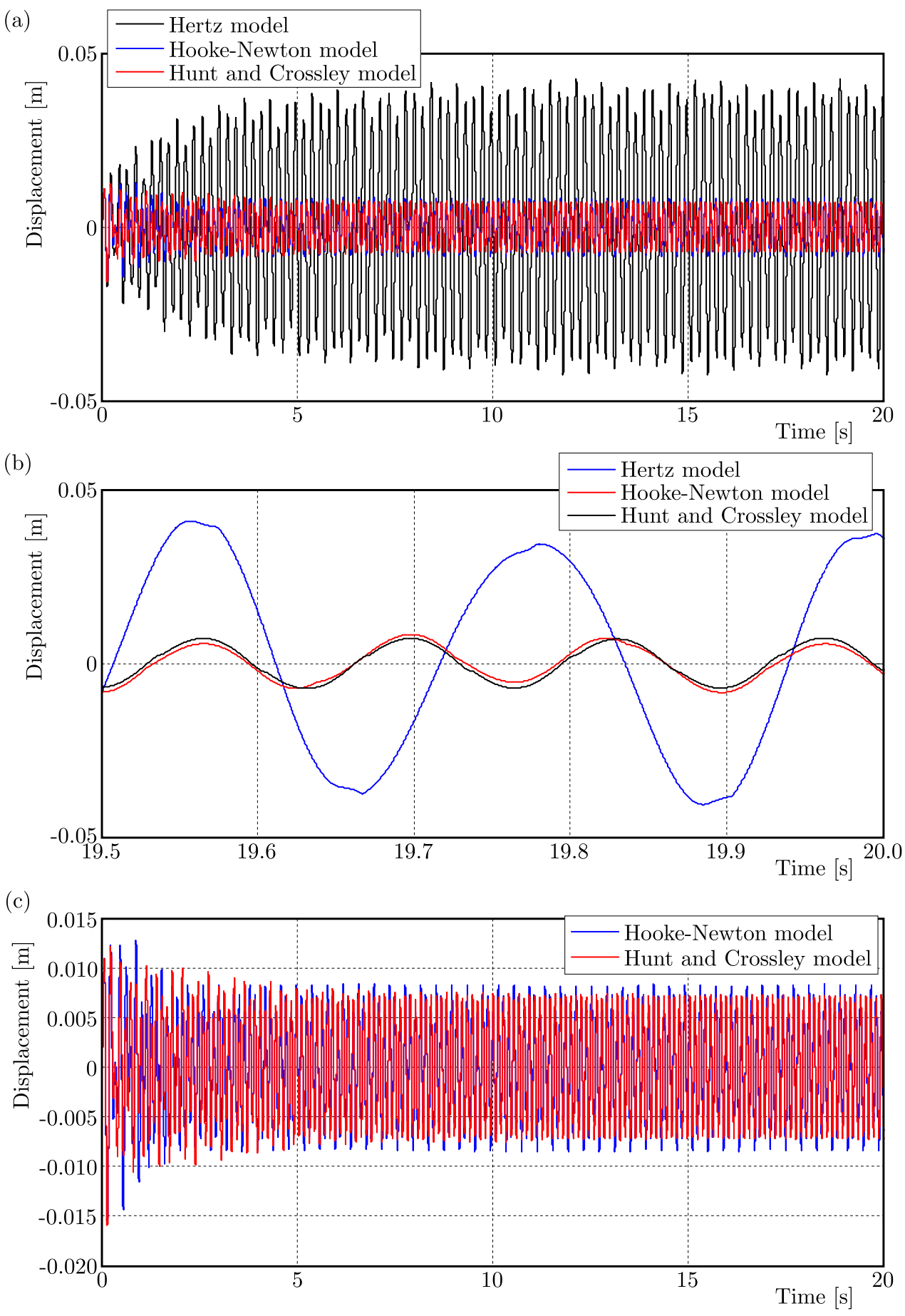

Fig. 7. System displacement for all collision models 
Once a mechanical system is equipped with a single unit impact absorber, accordingly to each model listed above and considering the same contact stiffness $\left(k_{1}=32600 \mathrm{~N} / \mathrm{m}^{3 / 2}\right)$ for all models for consistency, we note the following results:

- Regarding the displacement amplitudes of the system equipped with the absorber for all collision models, see Figs. 7a,b, the vibration amplitude is the lowest for the Hunt and Crossley model. Moreover, the response according to the Hooke-Newton model is closer to the Hunt and Crossley model response than the Hertz model one, Fig. 7c.

- Regarding the sensitivity to the restitution coefficient of the system displacement considering all collision models, Fig. 8, the Hunt and Crossley model is more sensitive to the impact quality than the Hooke-Newton model. Besides, when the restitution coefficient is equal to 1 (without absorber damping), the system maximum displacement is lower for the Hooke-Newton model relative to the Hertz and Hunt and Crossly models, which are similar. However, when the restitution coefficient is equal to 0.68 (with absorber damping), the system maximum displacement for the Hunt and Crossley model is the lowest.

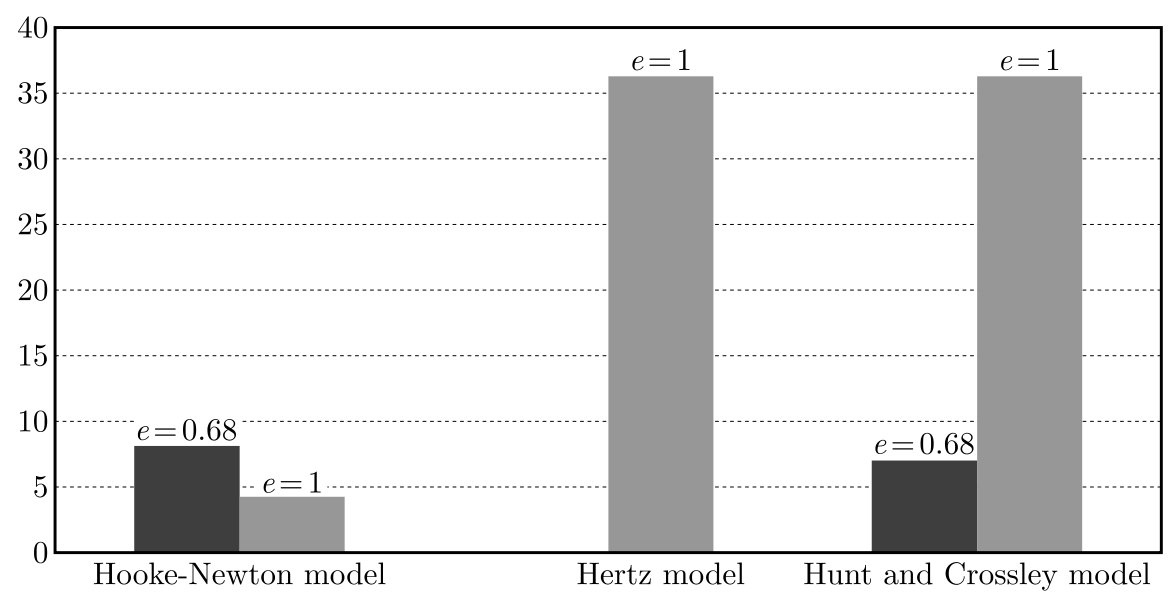

Fig. 8. System maximum displacement for all collision models

- Regarding the displacement attenuation of the system equipped with the absorber for all collision models, Fig. 9, both the Hooke-Newton model and the Hertz model correspond to an overestimation relative to the Hunt and Crossley model. However, the Hooke-Newton model displacement attenuation is closer to the Hunt and Crossley model displacement attenuation.

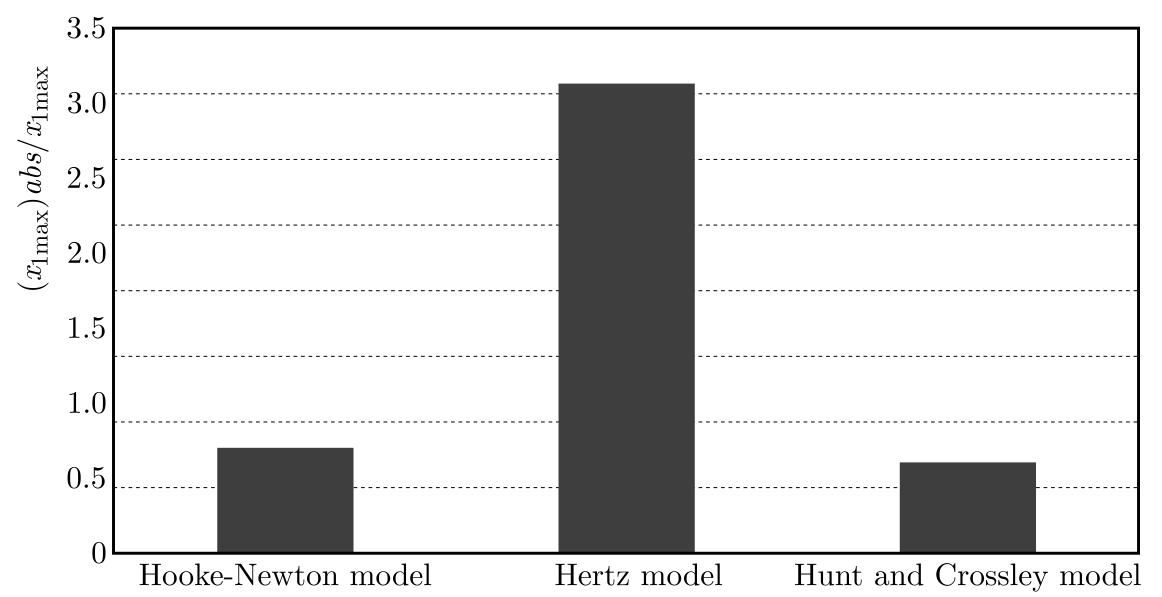

Fig. 9. System displacement attenuation for all collision models 
- Regarding the system dissipated energy for all collision models, Fig. 10, both the Hooke-Newton and Hertz models correspond to an underestimation relative to the Hunt and Crossley model. However, the Hooke-Newton model energy dissipation is higher than the Hertz model energy dissipation.
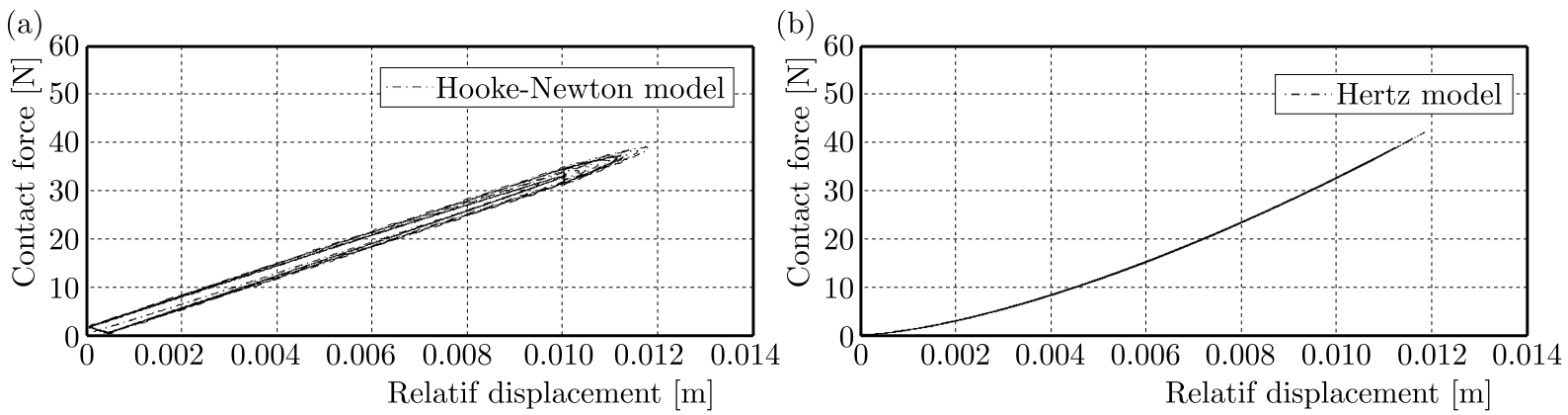

(c)

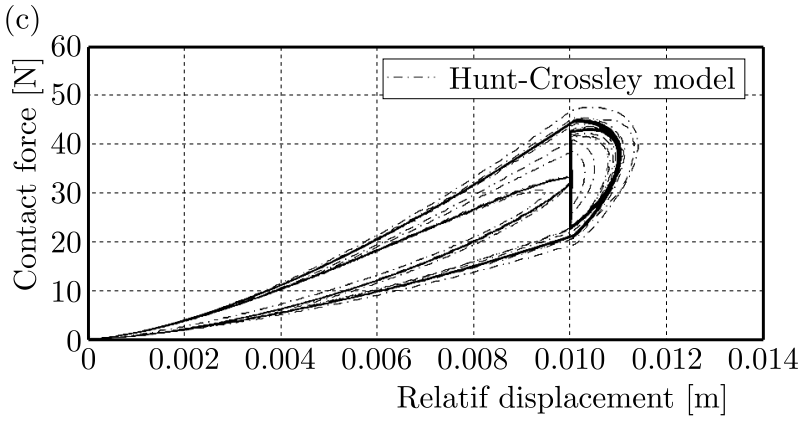

Fig. 10. Impact dissipated energy for all collision models: (a) Hooke-Newton model, (b) Hertz model, (c) Hunt and Crossley model

\section{Conclusions}

A single unit impact absorber is a ball absorber introduced in a mechanical system to attenuate its vibration with dissipating the vibratory energy through collisions between both bodies.

The effect of the collision modeling on the absorber performance was studied in this work. The viscoelastic linear model of Hooke-Newton, the elastic nonlinear model of Hertz and the viscoelastic nonlinear model of Hunt and Crossley were considered. For all cases, the Newmark method was used to integrate differential equations of motion considering the whole ball motion cycle: left impact-free motion-right impact. System responses were obtained for forced vibration, and a comparison was made to conclude on the absorber efficiency.

The comparison shows that the Hertz model overestimates the system displacement amplitude. Once the system is equipped with the absorber accordingly to the Hertz model, the main system vibration amplitudes increase instead of decreasing. The Hooke-Newton model is more realistic. The main system vibration amplitudes once equipped with the absorber decrease. The response, the displacement attenuation and the energy dissipation for the Hooke-Newton model are close to the Hunt and Crossley model, which is considered as the reference since it is more consistent with the notion of coefficient of restitution, commonly used to characterize energy losses during impacts.

Thus, in order to correctly model, analyze and simulate mechanical systems, particularly those including impact absorbers, appropriate contact force models must be adopted. 


\section{References}

1. Afsharfard A., Farshidianfar A., 2012, An efficient method to solve the strongly coupled nonlinear differential equations of impact dampers, Archive of Applied Mechanics, 82, 977-984

2. Alves J., Peixinho N., Da Silva M.T., Flores P., Lankarani H.M., 2015, A comparative study of the viscoelastic constitutive models for frictionless contact interfaces in solids, Mechanism and Machine Theory, 85, 172-188

3. Anagnostopoulos S.A., 2004, Equivalent viscous damping for modeling inelastic impacts in earthquake pounding problems, Earthquake Engineering and Structural Dynamics, 33, 897-902

4. Bapat C.N., Sankar S., 1985, Single unit impact damper in free and forced vibration, Journal of Sound and Vibration, 99, 85-94

5. Caserta A.J., Navarro H.A., Cabezas-Gomez L., 2016, Damping coefficient and contact duration relations for continuous nonlinear spring-dashpot contact model in DEM, Powder Technology, 302, 462-479

6. Cheng C.C., WAng J.Y., 2003, Free vibration analysis of a resilient impact damper, International Journal of Mechanical Sciences, 45, 589-604

7. Cheng J., Xu H., 2006, Inner mass impact damper for attenuating structure vibration, International Journal of Solids and Structures, 43, 5355-5369

8. Cheng J., Xu H., 2007, Periodic motions, bifurcation, and hysteresis of the vibro-impact system, Mechanics Based Design of Structures and Machines, 35, 179-203

9. Ema S., Marui E., 1996, Damping characteristics of an impact damper and its application, International Journal of Machine Tools and Manufacture, 36, 293-306

10. Gilardi G., Sharf I., 2002, Literature survey of contact dynamics modeling, Mechanism and Machine Theory, 37, 1213-1239

11. Grubin C., 1956, On the theory of the acceleration damper, Journal of Applied Mechanics, 23, 373-378

12. Hunt K.H., Crossley F.R.E., 1975, Coefficient of restitution interpreted as damping in vibroimpact, Journal of Applied Mechanics, 42, 440-445

13. Lieber P., Jensen D.P., 1945, An acceleration damper: development, design, and some applications, Transactions of ASME, 67, 523-530

14. Lu Z., Wang Z., Masri S.F., Lu X., 2017, Particle impact dampers: past, present, and future, Structural Control Health Monitoring, 25, 1-25

15. Marhadi K.S., Kinra V.K., 2005, Particle impact damping: effect of mass ratio, material and shape, Journal of Sound and Vibration, 283, 433-448 